\title{
Discrete Symmetries of Off-Shell Electromagnetism
}

\author{
Martin Land \\ Department of Computer Science \\ Hadassah College \\ P. O. Box 1114, Jerusalem 91010, Israel \\ email: martin@multinet.net.il
}

\begin{abstract}
This paper discusses the discrete symmetries of off-shell electromagnetism, the Stueckelberg-Schrodinger relativistic quantum theory and its associated 5D local gauge theory. Seeking a dynamical description of particle/antiparticle interactions, Stueckelberg developed a covariant mechanics with a monotonically increasing Poincaréinvariant parameter. In Stueckelberg's framework, worldlines are traced out through the parameterized evolution of spacetime events, which may advance or retreat with respect to the laboratory clock, depending on the sign of the energy, so that negative energy trajectories appear as antiparticles when the observer describes the evolution using the laboratory clock. The associated gauge theory describes local interactions between events (correlated by the invariant parameter) mediated by five off-shell gauge fields. These gauge fields are shown to transform tensorially under under space and time reflections - unlike the standard Maxwell fields - and the interacting quantum theory therefore remains manifestly Lorentz covariant. Charge conjugation symmetry in the quantum theory is achieved by simultaneous reflection of the sense of evolution and the fifth scalar field. Applying this procedure to the classical gauge theory leads to a purely classical manifestation of charge conjugation, placing the $C P T$ symmetries on the same footing in the classical and quantum domains. In the resulting picture, interactions do not distinguish between particle and antiparticle trajectories - charge conjugation merely describes the interpretation of observed negative energy trajectories according to the laboratory clock.
\end{abstract}

\section{Introduction}

\subsection{Stueckelberg's Model of Pair Creation/Annihilation}

In 1941, Stueckelberg [1] proposed a covariant Hamiltonian formalism for interacting spacetime events, in which the events evolve dynamically, as functions of a Poincaré invariant parameter (see also Fock [2]). In the classical mechanics, the particle worldline is traced out in terms of the values taken on by the four-vector $x^{\mu}(\tau)$ as the parameter proceeds monotonically from $\tau=-\infty$ to $\tau=\infty$. Stueckelberg's purpose was to ascribe pair creation/annihilation to a single worldline, generated dynamically by an event whose time 
coordinate advances or retreats with respect to the laboratory clock, as its instantaneous energy changes sign under interaction with a field. Figure 1 is a reconstruction of the corresponding illustration in Stueckelberg's paper [1].

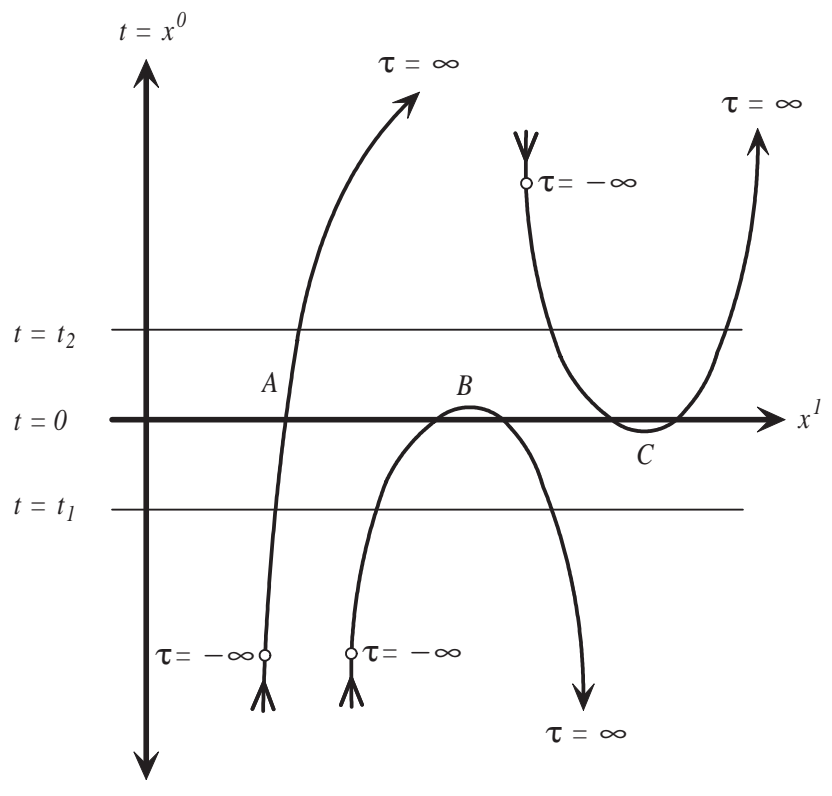

Figure 1: World Lines

A: Usual type, with a unique solution to $t(\tau)=x^{0}$ for each $x^{0}$

B: Annihilation type, with two solutions to $t(\tau)=x^{0}$ for $x^{0} \ll 0$ and no solution for $x^{0} \gg 0$

C: Creation type, with two solutions to $t(\tau)=x^{0}$ for $x^{0} \gg 0$ and no solution for $x^{0} \ll 0$

The invariant parameter $\tau$ is required because the worldlines of Figure 1 are generally not single-valued in spacetime. By regarding $\tau$ as a physical time parameter, Stueckelberg recognized two aspects of time [3], explicitly distinguishing the Einstein coordinate time $x^{0}$ from the temporal order $\tau$. Writing $\tau$-dependent equations of motion, Stueckelberg was led to a description of the antiparticle which is similar to Feynman's, but differs in its implementation of the discrete symmetries. In the standard treatment of CPT, based on Wigner's approach to time reversal [4], the $T$ operation is understood as both inversion of the time coordinate and reversal of motion - inversion of the temporal ordering of events. Despite the similarity of the worldlines in Figure 1 to Feynman's spacetime diagrams, the explicit parameterization of the curves by $\tau$ formally distinguishes the two aspects of time, and hence two notions of time reversal. Formal analysis of the dynamical framework arising from Stueckelberg's approach shows that, unlike the standard CPT implementation, fields and currents transform 
tensorially under the discrete Lorentz transformations. The charge conjugation operation is seen to connect laboratory observation with experiment, but does not play a role in interactions. Although $C P T$ invariance was not established as a fundamental symmetry until more than a decade after Stueckelberg and Feynman's initial work on this subject, and they did not utilize that approach, these symmetry properties expose the different interpretations of the antiparticle.

When standard textbooks (see for example [5]) discuss what is generally known as the Feynman-Stueckelberg approach, which represents antiparticles as negative energy modes propagating toward earlier times, they pose this model as an elegant field theoretic replacement for the older Dirac hole theory. However, Stueckelberg's paper addresses itself to a slightly different set of concerns. While hole theory predicted antiparticles in an attempt to solve the problem of negative energy solutions to the Dirac and Klein-Gordon equations, Stueckelberg's goal was the formulation of a relativistic generalization of classical and quantum mechanics ${ }^{1}$ capable of generating the curves of Figure 1. By 1941, pair creation and annihilation were regarded as experimentally observable phenomena, and the historical reasons they were first predicted (as an artifact of one or another candidate theory) were not necessarily relevant to their interpretation. Stueckelberg actually emphasized the negative energy trajectories as an advantage of his theory, not a problem to be solved. Since the parameter time $\tau$ is formally similar to the Galilean invariant time in Newtonian theory, this formalism served Stueckelberg's wider goal of generalizing the techniques of non-relativistic classical and quantum mechanics to covariant form.

While Stueckelberg defined classical and quantum states explicitly labeled by $\tau$, Feynman worked with quantum states, in which events are temporally ordered by algebraic segregation into initial states and final states. The Feynman prescription for the propagator is equivalent to Dyson's t-ordered product, which by exchanging particle creation and annihilation, can be understood as enforcing temporal ordering of in-events and out-events at the endpoints of a worldline. In discussing the path integral for the Klein-Gordon equation, Feynman observed [6] that explicitly labeling the temporal order of events by $\tau$ and assuming retarded

\footnotetext{
${ }^{1}$ Introducing his generalized Lorentz force, Stueckelberg suggests that the correct formulation of relativistic dynamics was not yet known, when he states, "La question se pose de savoir s'il est possible d'établir une méchanique covariante au sens d'Einstein, qui permette l'existence de telles courbes."
} 
propagation (with respect to $\tau$ ), leads to the $t$-ordering prescription for the propagator. However, we will see that the explicit labeling by $\tau$ affects the meanings of time reversal symmetry and charge conjugation.

Stueckelberg argued that pair annihilation is observed in worldlines of type B in Figure 1, in the fact that there are two solutions to $t(\tau)=t_{1}$, but no solution to $t(\tau)=t_{2}$. The observer will therefore first encounter two particle trajectories and then encounter none. That the magnitude of the electric charge should not change along the single worldline seems clear enough, but the identification of one part of the worldline as an antiparticle trajectory, further requires that the charge reverse sign. While this charge reflection may be grasped intuitively - carrying positive charge in one time direction is taken as equivalent to carrying negative charge in the opposite time direction - in standard field theory, charge conjugation is demonstrated through the action of the charge operator. In the parameterized formalism, the charge inversion appears directly at the classical level: as the event $x^{\mu}(\tau)$ evolves toward earlier values of $t=x^{0}$, the slope $d x^{0} / d \tau$ must become negative, and in Stueckelberg's generalized Lorentz force, this derivative multiplies the electric charge. Thus, particles and antiparticles do not appear as distinct classes of solutions to a defining equation, but as a single event whose qualitative behavior depends instantaneously on the dynamical value of its velocity.

\subsection{Gauge Theory}

To generalize the Lorentz equations, Stueckelberg proposed the covariant evolution equation

$$
\frac{d^{2} x^{\mu}}{d \tau^{2}}=-\Gamma_{\nu \rho}^{\mu} \frac{d x^{\nu}}{d \tau} \frac{d x^{\rho}}{d \tau}+e F^{\mu \nu} g_{\nu \rho} \frac{d x^{\rho}}{d \tau}+K^{\mu}
$$

in which the metric signature is $\operatorname{diag}(-,+,+,+)$ with index convention $\mu, \nu, \rho=0, \cdots, 3$, $F^{\mu \nu}$ is the electromagnetic field strength tensor, and $\Gamma_{\nu \rho}^{\mu}$ is an affine connection expressing the influence of gravitation. Stueckelberg observed that the mass

$$
m^{2}=-g_{\nu \rho} \frac{d x^{\nu}}{d \tau} \frac{d x^{\rho}}{d \tau}
$$


is a constant of integration for $K^{\mu}=0$. The proper time is found by scaling the invariant parameter through $d s= \pm \sqrt{d s^{2}}= \pm m d \tau$ so that when $K^{\mu}=0$,

$$
-g_{\nu \rho} \frac{d x^{\nu}}{m d \tau} \frac{d x^{\rho}}{m d \tau}=-\frac{d x^{\nu}}{d s} \frac{d x_{\nu}}{d s}=1
$$

Stueckelberg found no reason to claim the existence of the field $K^{\mu}$, without which the dynamical conservation of mass prevents the classical worldlines from entering the spacelike region, required for the transition from positive to negative energy. In the absence of $K^{\mu}$, Stueckelberg's equation may be derived from the classical Lagrangian

$$
L=\frac{1}{2} M \dot{x}^{\mu} \dot{x}_{\mu}+e \dot{x}^{\mu} A_{\mu}(x)
$$

and Euler-Lagrange equations

$$
\frac{d}{d \tau} \frac{\partial L}{\partial \dot{x}_{\mu}}-\frac{\partial L}{\partial x_{\mu}}=0
$$

where

$$
F^{\mu \nu}=\partial^{\mu} A^{\nu}-\partial^{\nu} A^{\mu}
$$

and we have introduced the constant parameter $M$ with dimension of mass. The equivalent flat space Hamiltonian formulation

$$
K=\frac{1}{2 M}\left(p^{\mu}-e A^{\mu}\right)\left(p_{\mu}-e A_{\mu}\right)
$$

with symplectic equations

$$
\frac{d x^{\mu}}{d \tau}=\dot{x}^{\mu}=\frac{\partial K}{\partial p_{\mu}} \quad \frac{d p^{\mu}}{d \tau}=\dot{p}^{\mu}=-\frac{\partial K}{\partial x_{\mu}}
$$

leads to a quantum theory defined by the equation,

$$
i \partial_{\tau} \psi(x, \tau)=\frac{1}{2 M}\left(p^{\mu}-e A^{\mu}\right)\left(p_{\mu}-e A_{\mu}\right) \psi(x, \tau)
$$

This quantum theory (see also [6]), enjoys the standard U(1) gauge invariance under local transformations of the type

$$
\begin{aligned}
\psi(x, \tau) & \longrightarrow \exp [i e \Lambda(x)] \psi(x, \tau) \\
A_{\mu} & \longrightarrow A_{\mu}+\partial_{\mu} \Lambda(x)
\end{aligned}
$$

but the global gauge invariance is associated with the five-dimensional conserved current

$$
\partial_{\mu} j^{\mu}+\partial_{\tau} \rho=0
$$


where

$$
\rho=|\psi(x, \tau)|^{2} \quad j^{\mu}=-\frac{i}{2 M}\left\{\psi^{*}\left(\partial^{\mu}-i e A^{\mu}\right) \psi-\psi\left(\partial^{\mu}+i e A^{\mu}\right) \psi^{*}\right\} .
$$

Stueckelberg [1] regarded (12) as a true current, leading to the interpretation of $|\psi(x, \tau)|^{2}$ as the probability density at $\tau$ of finding the event at the spacetime point $x$. However, under this interpretation, the non-zero divergence of the four-vector current $j^{\mu}(x, \tau)$ prevents its identification as the source of the $A^{\mu}(x)$. As a remedy, Stueckelberg observed that assuming $\rho \rightarrow 0$ pointwise as $\tau \rightarrow \pm \infty$, integration of (12) over $\tau$ leads to

$$
\partial_{\mu} J^{\mu}=0 \quad \text { where } \quad J^{\mu}(x)=\int_{-\infty}^{\infty} d \tau j^{\mu}(x, \tau) .
$$

However, in the resulting dynamical picture, the fields $A^{\mu}(x)$ which mediate particle interaction instantaneously at $\tau$, are induced by currents $J^{\mu}(x)$ whose support covers the particle worldlines, past and future. There is no a priori assurance that the particles moving in these Maxwell fields will trace out precisely the worldlines which induce the fields responsible for their motion.

In order to obtain a well-posed theory, Sa'ad, Horwitz, and Arshansky [19] introduced a $\tau$ dependent gauge field (see also [7]) and a fifth gauge compensation field, leading to a theory which differs in significant aspects from conventional electrodynamics, but whose zero modes coincide with the Maxwell theory. Writing $x^{5}=\tau$ and adopting the index convention

$$
\lambda, \mu, \nu=0,1,2,3 \quad \text { and } \quad \alpha, \beta, \gamma=0,1,2,3,5
$$

the Stueckelberg-Schrodinger equation

$$
\left(i \partial_{\tau}+e_{0} a_{5}\right) \psi(x, \tau)=\frac{1}{2 M}\left(p^{\mu}-e_{0} a^{\mu}\right)\left(p_{\mu}-e_{0} a_{\mu}\right) \psi(x, \tau)
$$

is invariant under the enlarged set of gauge transformations,

$$
\begin{aligned}
\psi(x, \tau) & \rightarrow e^{i e_{0} \Lambda(x, \tau)} \psi(x, \tau) \\
a_{\alpha}(x, \tau) & \rightarrow a_{\alpha}(x, \tau)+\partial_{\alpha} \Lambda(x, \tau)
\end{aligned}
$$

and admits the modified five dimensional conserved current

$$
\partial_{\alpha} j^{\alpha}=\partial_{\mu} j^{\mu}+\partial_{\tau} j^{5}=0
$$


where the probability density interpretation still holds for

$$
j^{5}=|\psi(x, \tau)|^{2}
$$

and the current becomes $\tau$-dependent through both the particle and the gauge fields

$$
j^{\mu}=\frac{-i}{2 M}\left[\psi^{*}\left(\partial^{\mu}-i e_{0} a^{\mu}\right) \psi-\psi\left(\partial^{\mu}+i e_{0} a^{\mu}\right) \psi^{*}\right] .
$$

The Stueckelberg-Schrodinger equation (15) may be derived by variation of the action

$$
\mathrm{S}=\int d^{4} x d \tau\left\{\psi^{*}\left(i \partial_{\tau}+e_{0} a_{5}\right) \psi-\frac{1}{2 M} \psi^{*}\left(p_{\mu}-e_{0} a_{\mu}\right)\left(p^{\mu}-e_{0} a^{\mu}\right) \psi-\frac{\lambda}{4} f_{\alpha \beta} f^{\alpha \beta}\right\}
$$

which includes a kinetic term for the fields, formed from the gauge invariant quantity

$$
f_{\alpha \beta}=\partial_{\alpha} a_{\beta}-\partial_{\beta} a_{\alpha}
$$

Sa'ad, et. al. formally raise the index $\beta=5$ in the term $f_{\mu 5}=\partial_{\mu} a_{5}-\partial_{\tau} a_{\mu}$ with the flat metric

$$
g^{\alpha \beta}=\operatorname{diag}(-1,1,1,1, \sigma), g^{55}=\sigma= \pm 1,
$$

corresponding to a $\mathrm{O}(4,1)$ or $\mathrm{O}(3,2)$ symmetry, which must break to $\mathrm{O}(3,1)$ in the presence of currents. Varying the action (21) with respect to the gauge fields, the equations of motion are found to be

$$
\partial_{\beta} f^{\alpha \beta}=\frac{e_{0}}{\lambda} j^{\alpha}=e j^{\alpha} \quad \epsilon^{\alpha \beta \gamma \delta \epsilon} \partial_{\alpha} f_{\beta \gamma}=0
$$

where $j^{\alpha}$ is given in (19) and (20). Although $\lambda$ and $e_{0}$ must be dimensional constants, the dimensionless ratio $e_{0} / \lambda$ is the Maxwell charge $e$. In four-vector component form, (24) becomes

$$
\begin{aligned}
& \partial_{\nu} f^{\mu \nu}-\partial_{\tau} f^{5 \mu}=e j^{\mu} \quad \partial_{\mu} f^{5 \mu}=e \rho . \\
& \partial_{\mu} f_{\nu \rho}+\partial_{\nu} f_{\rho \mu}+\partial_{\rho} f_{\mu \nu}=0 \quad \partial_{\mu} f_{5 \nu}-\partial_{\nu} f_{5 \mu}-\partial_{\tau} f_{\mu \nu}=0,
\end{aligned}
$$

which may be seen as a four-dimensional analog of the three-vector Maxwell equations in the usual form,

$$
\begin{array}{lr}
\nabla \times \mathbf{H}-\partial_{0} \mathbf{E}=e \mathbf{J} & \nabla \cdot \mathbf{E}=e J^{0} \\
\nabla \cdot \mathbf{H}=0 & \nabla \times \mathbf{E}+\partial_{0} \mathbf{H}=0 .
\end{array}
$$


The three vector form of the field equations [8], defined through

$$
\begin{gathered}
e_{i}=f^{0 i} \quad h_{i}=\epsilon_{i j k} f^{j k} \\
\epsilon^{i}=f^{5 i} \quad \epsilon^{0}=f^{50}
\end{gathered}
$$

is useful for the study of the discrete symmetries. These field equations (generalizations of (27) and (28)) are

$$
\begin{gathered}
\nabla \cdot \mathbf{e}-\partial_{\tau} \epsilon^{0}=e j^{0} \quad \nabla \times \mathbf{e}+\partial_{0} \mathbf{h}=0 \\
\nabla \times \mathbf{h}-\partial_{0} \mathbf{e}-\partial_{\tau} \epsilon=e \mathbf{j} \quad \nabla \cdot \mathbf{h}=0 \\
\nabla \cdot \epsilon+\partial_{0} \epsilon^{0}=e j^{5} \quad \nabla \times \epsilon-\sigma \partial_{\tau} \mathbf{h}=0 \\
\nabla \epsilon^{0}+\sigma \partial_{\tau} \mathbf{e}+\partial_{0} \epsilon=0 .
\end{gathered}
$$

\subsubsection{Concatenation}

The connection with Maxwell theory enlarges on Stueckelberg's observation in (13). Under the conditions $j^{5} \rightarrow 0$ and $f^{5 \mu} \rightarrow 0$, pointwise in $x$ as $\tau \rightarrow \pm \infty$, integration of (24) over $\tau$, called concatenation of events into a worldlines [9], recovers the relations

$$
\partial_{\nu} F^{\mu \nu}=e J^{\mu} \quad \epsilon^{\mu \nu \rho \lambda} \partial_{\mu} F_{\nu \rho}=0
$$

where

$$
F^{\mu \nu}(x)=\int_{-\infty}^{\infty} d \tau f^{\mu \nu}(x, \tau) \quad \text { and } \quad A^{\mu}(x)=\int_{-\infty}^{\infty} d \tau a^{\mu}(x, \tau)
$$

and so $a^{\alpha}(x, \tau)$ has been called the pre-Maxwell field. It follows from (36) that $e_{0}$ and $\lambda$ have dimensions of length. 


\subsubsection{Implications for the Parameterized Mechanics}

As in the non-relativistic case, the two-body action-at-a-distance potential in the HorwitzPiron theory [10] may be understood as the approximation $-e_{0} a_{5}(x, \tau) \longrightarrow V(x)$. Within this framework, solutions have been found for the generalizations of the standard central force problem, including potential scattering [11] and bound states [12, 13]. Examination of radiative transitions [14, in particular the Zeeman [15] and Stark effects [16], indicate that all five components of the gauge potential are necessary for an adequate explanation of observed phenomenology.

\subsubsection{The Classical Lorentz Force}

From the quantum Hamiltonian in (15) one is led to the classical Lagrangian

$$
L=\dot{x}^{\mu} p_{\mu}-K=\frac{1}{2} M \dot{x}^{\mu} \dot{x}_{\mu}+e_{0} \dot{x}^{\alpha} a_{\alpha}=\frac{1}{2} M \dot{x}^{\mu} \dot{x}_{\mu}+e_{0} \dot{x}^{\mu} a_{\mu}+e_{0} a_{5}
$$

and, under variation with respect to $x^{\mu}$, the Lorentz force [8]

$$
M \ddot{x}^{\mu}=e_{0} f_{\alpha}^{\mu}(x, \tau) \dot{x}^{\alpha}=e_{0}\left[f^{\mu}{ }_{\nu}(x, \tau) \dot{x}^{\nu}+f^{\mu}{ }_{5}(x, \tau)\right]
$$

where $f_{\alpha \beta}$ is the gauge invariant quantity (22). The field strength $f^{5 \mu}$ plays the role of Stueckelburg's field $K^{\mu}$, and so the enlarged gauge symmetry is seen to provide a consistent basis for this additional interaction. The effect of this interaction on rest mass, as Stueckelburg found earlier,

$$
\frac{d}{d \tau}\left(-\frac{1}{2} M \dot{x}^{2}\right)=-M \dot{x}^{\mu} \ddot{x}_{\mu}=-e_{0} \dot{x}^{\mu}\left(f_{\mu 5}+f_{\mu \nu} \dot{x}^{\nu}\right)=-e_{0} \dot{x}^{\mu} f_{\mu 5}=e_{0} \sigma f_{\alpha}^{5} \dot{x}^{\alpha}
$$

appears formally as the "fifth" component of the Lorentz force law. Conservation of mass, $\dot{x}^{2}=$ constant, requires that

$$
f_{5 \mu}=0 \quad \text { and } \quad \partial_{\tau} f^{\mu \nu}=0
$$

where the second condition follows from (26) for $f^{5 \mu}=0$. The generalization of (38) to curved spacetime was found [17] to be

$$
M\left[\ddot{x}^{\mu}+\Gamma^{\mu \lambda \nu} \dot{x}_{\lambda} \dot{x}_{\nu}\right]=e_{0} f_{\alpha}^{\mu}(x, \tau) \dot{x}^{\alpha}=e_{0}\left[f_{\nu}^{\mu}(x, \tau) \dot{x}^{\nu}+f^{\mu}{ }_{5}(x, \tau)\right]
$$


with

$$
\Gamma^{\mu \lambda \nu}=-\frac{1}{2}\left(\partial^{\nu} g^{\lambda \mu}+\partial^{\lambda} g^{\mu \nu}-\partial^{\mu} g^{\lambda \nu}\right)
$$

Equation (41) can be identified with Stueckelberg's proposed equation (11). This expression was shown [17] to be the most general expression for a classical force consistent with the quantum commutation relations

$$
\left[x^{\mu}, x^{\nu}\right]=0 \quad m\left[x^{\mu}, \dot{x}^{\nu}\right]=-i \hbar g^{\mu \nu}(x)
$$

Relaxing the mass-shell constraint in (43) breaks general reparameterization invariance in (37), but, under the conditions (40), the remaining $\tau$-translation symmetry is associated, via Noether's theorem, with dynamic conservation of the mass. It has been shown [8] that while the material events and gauge fields may exchange mass when the conditions (40) do not hold, the total mass-energy of the particles and fields is conserved. Since the gauge fields propagate with a mass spectrum, this theory has been called off-shell electrodynamics.

\subsubsection{Classical Coulomb Problem}

Further questions of interpretation of the five dimensional formalism arise in treating the classical Coulomb problem. Posing the classical equations of motion for a test event (worldline $B$ in Figure 2) moving in the field induced by a 'static' event (worldline $A$ in Figure 2) evolving uniformly along the time axis, one is faced with three interrelated problems. 


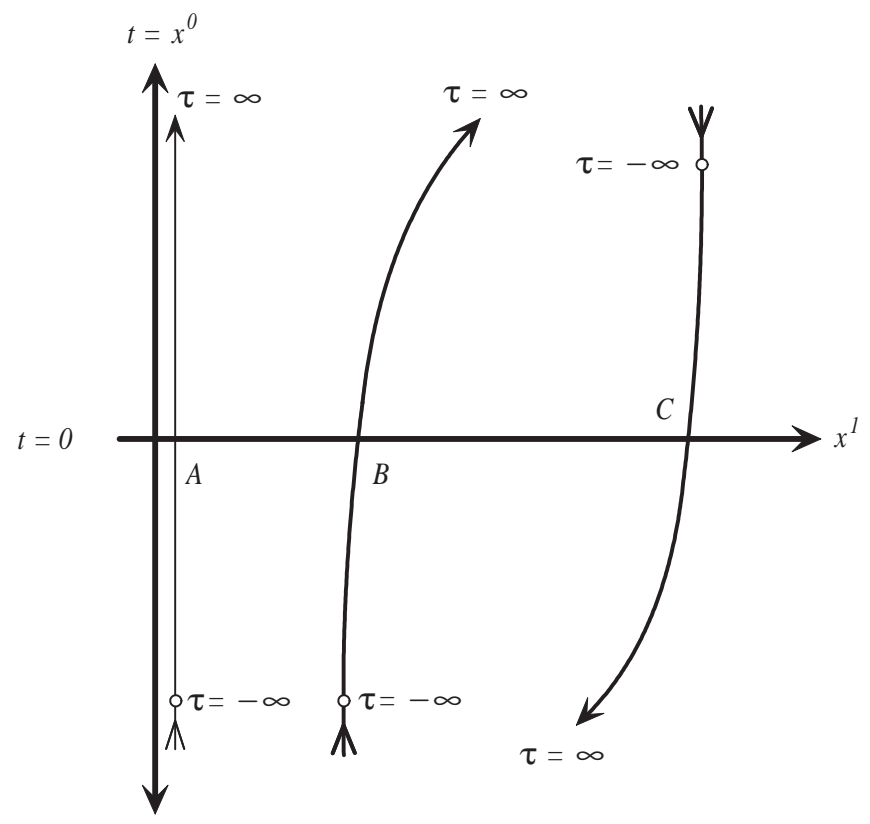

Figure 2: Elastic Scattering

A: 'Static' event evolving uniformly along the time axis

B: Test event with $\dot{x}^{0}(-\infty)=1$ evolving in field induced by A

C: Test event with $\dot{x}^{0}(-\infty)=-1$ evolving in field induced by $\mathrm{A}$

First, the classical current density of the point events involves a delta function centered on the event,

$$
j^{0}(x, \tau)=\delta\left(x^{0}-\tau\right) \delta^{3}(\mathbf{x}) .
$$

Second, the structure of the Green's function [18,

$$
G(x, \tau)=-\frac{1}{4 \pi} \delta\left(x^{2}\right) \delta(\tau)-\frac{1}{2 \pi^{2}} \frac{\partial}{\partial x^{2}} \frac{\theta\left(-\sigma g_{\alpha \beta} x^{\alpha} x^{\beta}\right)}{\sqrt{-\sigma g_{\alpha \beta} x^{\alpha} x^{\beta}}}
$$

for the five dimensional wave equation [19]

$$
\partial_{\alpha} \partial^{\alpha} f^{\beta \gamma}=\left(\partial_{\mu} \partial^{\mu}+\partial_{\tau} \partial^{\tau}\right) f^{\beta \gamma}=\left(\partial_{\mu} \partial^{\mu}+\sigma \partial_{\tau}^{2}\right) f^{\beta \gamma}=-e\left(\partial^{\beta} j^{\gamma}-\partial^{\gamma} j^{\beta}\right)
$$

carries these delta functions into the induced gauge potentials. Third, the $\tau$-translation symmetry of the asymptotic events leads to a strong dependence on initial conditions that, under concatenation, should not be observable; that is, scattering in the gauge field induced by the event $x^{\mu}(\tau-a)$ will depend qualitatively on the value of $a$, even though the concatenated Coulomb field does not. A reasonable approach was found [20] by smoothing the 
field-inducing current as

$$
j_{\varphi}^{\alpha}(x, \tau)=\int_{-\infty}^{\infty} d s \varphi(\tau-s) j^{\alpha}(x, s)
$$

where $\varphi(\tau)$ is the Laplace distribution

$$
\varphi(\tau)=\frac{1}{2 \lambda} e^{-|\tau| / \lambda}
$$

Since the smoothing distribution satisfies

$$
\int_{-\infty}^{\infty} d \tau \varphi(\tau)=1
$$

the concatenated Maxwell current is not affected by the integration (47). This approach leads, in the low energy case, to a classical Yukawa potential,

$$
\begin{gathered}
a_{\varphi}^{0}(x, \tau)=\frac{1}{2 \lambda}\left[-\frac{e}{4 \pi R} e^{-R / \lambda}\right]\left[\frac{1}{2}\left(\frac{d x^{0}}{d \tau}+1\right)\right] \simeq \frac{1}{2 \lambda}\left[-\frac{e}{4 \pi R} e^{-R / \lambda}\right], \\
M \frac{d^{2} \mathbf{x}}{d \tau^{2}} \simeq 2 \lambda \text { e } \nabla a_{\varphi}^{0}(x, \tau)=\nabla\left[-\frac{e^{2}}{4 \pi R} e^{-R / \lambda}\right],
\end{gathered}
$$

with interpretation of $\lambda$ as a cut-off in the mass spectrum of the photons mediating the interaction. It was shown in 21] that replacing the usual kinetic term for the electromagnetic field strengths in (21)

$$
S_{\text {em-kinetic }}^{0}=\frac{\lambda}{4} \int d^{4} x d \tau f^{\alpha \beta}(x, \tau) f_{\alpha \beta}(x, \tau)
$$

with the higher-order derivative term

$$
S_{\text {em-kinetic }}=S_{\text {em-kinetic }}^{0}+\frac{\lambda^{3}}{4} \int d^{4} x d \tau\left(\partial_{\tau} f^{\alpha \beta}(x, \tau)\right)\left(\partial_{\tau} f_{\alpha \beta}(x, \tau)\right)
$$

is equivalent to the ad hoc approach taken in (47). This approach puts the theory into a form amenable to quantization and provides the mass cut-off which makes the field theory finite at all orders of perturbation theory.

\subsection{Particle/Antiparticle Problem}

By Stueckelberg's interpretation of the worldlines, application of the classical Coulomb case to elastic particle-antiparticle scattering seems only to require that the initial conditions 
of the test event include $d x^{0} / d \tau \leq 0$ (worldline $C$ in Figure 2 shows an event evolving from initial condition $t=-\tau$ ). However, in two aspects, the solution to the scattering problem posed in this way seems inadequate. First, we notice that in the low energy case, $d x^{0} / d \tau \simeq-1$, the Lorentz force (50) takes the form

$$
a_{\varphi}^{0}(x, \tau)=\frac{1}{2 \lambda}\left[-\frac{e}{4 \pi R} e^{-R / \lambda}\right]\left[\frac{1}{2}\left(\frac{d x^{0}}{d \tau}+1\right)\right] \simeq 0
$$

and it appears that no scattering takes place. Second, in modern treatments (for example [22, 5]), based on the CPT theorem, the antiparticle is characterized as a particle with the signs of all its additive quantum numbers reversed. Following Wigner, the quantum reflection $\theta$, which both reverses the spacetime parameters and exchanges the past and future, is an antiunitary operator. Since $\theta$ must be independent of any internal symmetries associated with generators $\left\{F_{n}\right\}$, the antiunitary of $\theta$ requires that

$$
\theta e^{i F_{n} \alpha^{n}} \theta^{-1}=e^{i F_{n} \alpha^{n}} \Rightarrow \theta\left[i F_{\mu}\right] \theta^{-1}=-i\left[\theta F_{\mu} \theta^{-1}\right] \quad \Rightarrow \quad \theta F_{\mu} \theta^{-1}=-F_{\mu}
$$

and so all additive quantum numbers change sign for the antiparticle. However, in the Stueckelberg theory, states are labeled by the parameter $\tau$, which is not affected by spacetime inversion, and the argument is not immediately applicable to the event evolving toward earlier $t$. It is not clear how this general reflection of internal symmetries is related to the dynamic evolution of the energy. For these reasons, we are led to reconsider whether the antiparticle differs from the particle only in the sign of $d x^{0} / d \tau$. In this paper, we address these issues by examining the discrete symmetries in a formal manner.

\section{Discrete Symmetries of Off-Shell Electrodynamics}

We take the improper Lorentz transformations, in the passive sense, as reversing the orientation of the space and time axes. This approach is similar to the standard approach, but differs from the non-relativistic formulation following Wigner [4] (see also [23]), in which time reversal changes the sign of velocities, momenta, and the space part of currents. Since velocities are $\tau$-derivatives, they transform tensorially under the improper Lorentz transformations, and so time and space inversion are handled as coordinates on the same footing. Reversal of the sense of motion, which is part of time reversal in the standard treatment, 
would be associated with $\tau$-reversal in this theory. However, since $\tau$-reversal cannot be implemented as a Lorentz transformation, we do not assume that the theory must be form covariant under such an operation.

Then space inversion acts as

$$
x=\left(x^{0}, \mathbf{x}\right) \underset{P}{\longrightarrow} x_{P}=\left(x_{P}^{0}, \mathbf{x}_{P}\right)=\left(x^{0},-\mathbf{x}\right)
$$

and time inversion acts as

$$
x=\left(x^{0}, \mathbf{x}\right) \underset{T}{\longrightarrow} x_{T}=\left(x_{T}^{0}, \mathbf{x}_{T}\right)=\left(-x^{0}, \mathbf{x}\right) .
$$

From the conventional observation that electromagnetic interactions are independent of the orientation of reference frames, we expect that the pre-Maxwell equations expressed in the coordinates $x_{P}$ or $x_{T}$ will be identical in form to the equations expressed in $x$ coordinates. In order to clarify our method, we take a familiar example from Maxwell theory, where form invariance of the Coulomb law implies that

$$
\nabla \cdot \mathbf{E}(t, \mathbf{x})=\rho(t, \mathbf{x}) \underset{P}{\longrightarrow} \nabla_{P} \cdot \mathbf{E}_{P}\left(t_{P}, \mathbf{x}_{P}\right)=\rho_{P}\left(t_{P}, \mathbf{x}_{P}\right) .
$$

Since $\rho(t, \mathbf{x})$ is a scalar field on spacetime, we expect that its value at the point $(t, \mathbf{x})$ equals the value of the transformed field $\rho_{P}$ at the corresponding point $\left(t_{P}, \mathbf{x}_{P}\right)$, that is

$$
\rho_{P}\left(t_{P}, \mathbf{x}_{P}\right)=\rho(t, \mathbf{x})
$$

Therefore,

$$
\begin{aligned}
\nabla_{P} \cdot \mathbf{E}_{P}\left(t_{P}, \mathbf{x}_{P}\right) & =\rho(t, \mathbf{x}) \\
-\nabla \cdot \mathbf{E}_{P}(t,-\mathbf{x}) & =\rho(t, \mathbf{x}) \\
\nabla \cdot\left\{-\mathbf{E}_{P}(t,-\mathbf{x})\right\} & =\rho(t, \mathbf{x}) .
\end{aligned}
$$

Comparing (62) with (58) we notice that $\mathbf{E}(t, \mathbf{x})$ and $-\mathbf{E}_{P}(t,-\mathbf{x})$ satisfy the same equation, so conclude

$$
\mathbf{E}(t, \mathbf{x})=-\mathbf{E}_{P}(t,-\mathbf{x})=-\mathbf{E}_{P}\left(t_{P}, \mathbf{x}_{P}\right)
$$

Standard treatments of the space and time reversal properties of the Maxwell theory (see for example [23]) study the field equations under the assumption of orientation invariance. However, since pre-Maxwell electrodynamics emerged from the requirement of local gauge invariance, from which the classical Lorentz force follows directly, it is more appropriate to begin with (38). 


\subsection{Space Inversion}

Under $P$, the pre-Maxwell Lorentz equations in three-vector form

$$
\begin{aligned}
M \frac{d^{2} x^{0}}{d \tau^{2}} & =e_{0}\left[\mathbf{e}(t, \mathbf{x}, \tau) \cdot \frac{d \mathbf{x}}{d \tau}-\sigma \epsilon^{0}(t, \mathbf{x}, \tau)\right] \\
M \frac{d^{2} \mathbf{x}}{d \tau^{2}} & =e_{0}\left[\mathbf{e}(t, \mathbf{x}, \tau) \frac{d x^{0}}{d \tau}+\frac{d \mathbf{x}}{d \tau} \times \mathbf{h}(t, \mathbf{x}, \tau)-\sigma \epsilon(t, \mathbf{x}, \tau)\right]
\end{aligned}
$$

become

$$
\begin{aligned}
M \frac{d^{2} x_{P}^{0}}{d \tau^{2}} & =e_{0}\left[\mathbf{e}_{P}\left(t_{P}, \mathbf{x}_{P}, \tau\right) \cdot \frac{d \mathbf{x}_{P}}{d \tau}-\sigma \epsilon_{P}^{0}\left(t_{P}, \mathbf{x}_{P}, \tau\right)\right] \\
M \frac{d^{2} \mathbf{x}_{P}}{d \tau^{2}} & =e_{0}\left[\mathbf{e}_{P}\left(t_{P}, \mathbf{x}_{P}, \tau\right) \frac{d x_{P}^{0}}{d \tau}+\frac{d \mathbf{x}_{P}}{d \tau} \times \mathbf{h}_{P}\left(t_{P}, \mathbf{x}_{P}, \tau\right)-\sigma \epsilon_{P}\left(t_{P}, \mathbf{x}_{P}, \tau\right)\right]
\end{aligned}
$$

so that

$$
\begin{aligned}
M \frac{d^{2} x^{0}}{d \tau^{2}} & =e_{0}\left[\mathbf{e}_{P}\left(t_{P}, \mathbf{x}_{P}, \tau\right) \cdot\left(-\frac{d \mathbf{x}}{d \tau}\right)-\sigma \epsilon_{P}^{0}\left(t_{P}, \mathbf{x}_{P}, \tau\right)\right] \\
-M \frac{d^{2} \mathbf{x}}{d \tau^{2}} & =e_{0}\left[\mathbf{e}_{P}\left(t_{P}, \mathbf{x}_{P}, \tau\right) \frac{d x^{0}}{d \tau}-\frac{d \mathbf{x}}{d \tau} \times \mathbf{h}_{P}\left(t_{P}, \mathbf{x}_{P}, \tau\right)-\sigma \epsilon_{P}\left(t_{P}, \mathbf{x}_{P}, \tau\right)\right]
\end{aligned}
$$

and finally

$$
\begin{aligned}
M \frac{d^{2} x^{0}}{d \tau^{2}} & =e_{0}\left[\left(-\mathbf{e}_{P}\left(t_{P}, \mathbf{x}_{P}, \tau\right)\right) \cdot \frac{d \mathbf{x}}{d \tau}-\sigma\left(\epsilon_{P}^{0}\left(t_{P}, \mathbf{x}_{P}, \tau\right)\right)\right] \\
M \frac{d^{2} \mathbf{x}}{d \tau^{2}} & =e_{0}\left[\left(-\mathbf{e}_{P}\left(t_{P}, \mathbf{x}_{P}, \tau\right)\right) \frac{d x^{0}}{d \tau}+\frac{d \mathbf{x}}{d \tau} \times\left(\mathbf{h}_{P}\left(t_{P}, \mathbf{x}_{P}, \tau\right)\right)-\sigma\left(-\epsilon\left(t_{P}, \mathbf{x}_{P}, \tau\right)\right)\right]
\end{aligned}
$$

Comparing (64) and (65) with (70) and (171) shows that

$$
\begin{aligned}
\mathbf{e}_{P}\left(t_{P}, \mathbf{x}_{P}, \tau\right) & =-\mathbf{e}(t, \mathbf{x}, \tau) \\
\mathbf{h}_{P}\left(t_{P}, \mathbf{x}_{P}, \tau\right) & =\mathbf{h}(t, \mathbf{x}, \tau) \\
\epsilon_{P}^{0}\left(t_{P}, \mathbf{x}_{P}, \tau\right) & =\epsilon^{0}(t, \mathbf{x}, \tau) \\
\epsilon_{P}\left(t_{P}, \mathbf{x}_{P}, \tau\right) & =-\epsilon(t, \mathbf{x}, \tau)
\end{aligned}
$$

and we recognize (72) and (73) as the behavior of the Maxwell electric and magnetic 3-vectors under parity. 


\subsection{Time Inversion}

Under $T$, (64) and (65) become

$$
\begin{aligned}
M \frac{d^{2} x_{T}^{0}}{d \tau^{2}} & =e_{0}\left[\mathbf{e}_{T}\left(t_{T}, \mathbf{x}_{T}, \tau\right) \cdot \frac{d \mathbf{x}_{T}}{d \tau}-\sigma \epsilon_{T}^{0}\left(t_{T}, \mathbf{x}_{T}, \tau\right)\right] \\
M \frac{d^{2} \mathbf{x}_{T}}{d \tau^{2}} & =e_{0}\left[\mathbf{e}_{T}\left(t_{T}, \mathbf{x}_{T}, \tau\right) \frac{d x_{T}^{0}}{d \tau}+\frac{d \mathbf{x}_{T}}{d \tau} \times \mathbf{h}_{T}\left(t_{T}, \mathbf{x}_{T}, \tau\right)-\sigma \epsilon_{T}\left(t_{T}, \mathbf{x}_{T}, \tau\right)\right]
\end{aligned}
$$

so that

$$
\begin{aligned}
-M \frac{d^{2} x^{0}}{d \tau^{2}} & =e_{0}\left[\mathbf{e}_{T}\left(t_{T}, \mathbf{x}_{T}, \tau\right) \cdot \frac{d \mathbf{x}}{d \tau}-\sigma \epsilon_{T}^{0}\left(t_{T}, \mathbf{x}_{T}, \tau\right)\right] \\
M \frac{d^{2} \mathbf{x}}{d \tau^{2}} & =e_{0}\left[\mathbf{e}_{T}\left(t_{T}, \mathbf{x}_{T}, \tau\right)\left(-\frac{d x^{0}}{d \tau}\right)+\frac{d \mathbf{x}}{d \tau} \times \mathbf{h}_{T}\left(t_{T}, \mathbf{x}_{T}, \tau\right)-\sigma \epsilon_{T}\left(t_{T}, \mathbf{x}_{T}, \tau\right)\right]
\end{aligned}
$$

and finally

$$
\begin{aligned}
& M \frac{d^{2} x^{0}}{d \tau^{2}}=e_{0}\left[\left(-\mathbf{e}_{T}\left(t_{T}, \mathbf{x}_{T}, \tau\right)\right) \cdot \frac{d \mathbf{x}}{d \tau}-\sigma\left(-\epsilon_{T}^{0}\left(t_{T}, \mathbf{x}_{T}, \tau\right)\right)\right] \\
& M \frac{d^{2} \mathbf{x}}{d \tau^{2}}=e_{0}\left[\left(-\mathbf{e}_{T}\left(t_{T}, \mathbf{x}_{T}, \tau\right)\right) \frac{d x^{0}}{d \tau}+\frac{d \mathbf{x}}{d \tau} \times\left(\mathbf{h}_{T}\left(t_{T}, \mathbf{x}_{T}, \tau\right)\right)-\sigma\left(\epsilon_{T}\left(t_{T}, \mathbf{x}_{T}, \tau\right)\right)\right]
\end{aligned}
$$

Comparing (64) and (65) with (70) and (71) shows that

$$
\begin{aligned}
\mathbf{e}_{T}\left(t_{T}, \mathbf{x}_{T}, \tau\right) & =-\mathbf{e}(t, \mathbf{x}, \tau) \\
\mathbf{h}_{T}\left(t_{T}, \mathbf{x}_{T}, \tau\right) & =\mathbf{h}(t, \mathbf{x}, \tau) \\
\epsilon_{T}^{0}\left(t_{T}, \mathbf{x}_{T}, \tau\right) & =-\epsilon^{0}(t, \mathbf{x}, \tau) \\
\epsilon_{T}\left(t_{T}, \mathbf{x}_{T}, \tau\right) & =\epsilon(t, \mathbf{x}, \tau)
\end{aligned}
$$

and here we notice that (82) and (83) are opposite to the standard behavior of the electric and magnetic 3-vectors under time inversion. This opposite behavior can be attributed to our having respected the independence of $x^{0}(\tau)$ as a function of $\tau$, not constrained by the mass-shell condition

$$
\frac{d x^{0}}{d \tau}=+\frac{1}{\sqrt{1-(d \mathbf{x} / d t)^{2}}}
$$




\subsection{Currents}

The pre-Maxwell equations in 3-vector form are

$$
\begin{aligned}
\nabla \cdot \mathbf{e}-\partial_{\tau} \epsilon^{0} & =e j^{0} \\
\nabla \times \mathbf{h}-\partial_{0} \mathbf{e}-\partial_{\tau} \epsilon & =e \mathbf{j} \\
\nabla \cdot \epsilon+\partial_{0} \epsilon^{0} & =e j^{5} \\
\nabla \cdot \mathbf{h} & =0 \\
\nabla \times \mathbf{e}+\partial_{0} \mathbf{h} & =0 \\
\nabla \times \epsilon-\sigma \partial_{\tau} \mathbf{h} & =0 \\
\nabla \epsilon^{0}+\sigma \partial_{\tau} \mathbf{e}+\partial_{0} \epsilon & =0
\end{aligned}
$$

so that under space inversion $P$, they become

$$
\begin{aligned}
(-\nabla) \cdot(-\mathbf{e})-\partial_{\tau} \epsilon^{0} & =\nabla \cdot \mathbf{e}-\partial_{\tau} \epsilon^{0}=e j_{P}^{0} \\
(-\nabla) \times \mathbf{h}-\partial_{0}(-\mathbf{e})-\partial_{\tau}(-\epsilon) & =-\left[\nabla \times \mathbf{h}-\partial_{0} \mathbf{e}-\partial_{\tau} \epsilon\right]=e \mathbf{j}_{P} \\
(-\nabla) \cdot(-\epsilon)+\partial_{0} \epsilon^{0} & =\nabla \cdot \epsilon+\partial_{0} \epsilon^{0}=e j_{P}^{5} \\
(-\nabla) \cdot \mathbf{h} & =-[\nabla \cdot \mathbf{h}]=0 \\
(-\nabla) \times(-\mathbf{e})+\partial_{0} \mathbf{h} & =\nabla \times \mathbf{e}+\partial_{0} \mathbf{h}=0 \\
(-\nabla) \times(-\epsilon)-\sigma \partial_{\tau} \mathbf{h} & =\nabla \times \epsilon-\sigma \partial_{\tau} \mathbf{h}=0 \\
(-\nabla) \epsilon^{0}+\sigma \partial_{\tau}(-\mathbf{e})+\partial_{0}(-\epsilon) & =-\left[\nabla \epsilon^{0}+\sigma \partial_{\tau} \mathbf{e}+\partial_{0} \epsilon\right]=0
\end{aligned}
$$

which are form invariant under the choices

$$
\begin{aligned}
& j_{P}^{0}\left(t_{P}, \mathbf{x}_{P}, \tau\right)=j^{0}(t, \mathbf{x}, \tau) \\
& \mathbf{j}_{P}\left(t_{P}, \mathbf{x}_{P}, \tau\right)=-\mathbf{j}(t, \mathbf{x}, \tau) \\
& j_{P}^{5}\left(t_{P}, \mathbf{x}_{P}, \tau\right)=j^{5}(t, \mathbf{x}, \tau)
\end{aligned}
$$


Similarly, under $T$,

$$
\begin{aligned}
\nabla \cdot(-\mathbf{e})-\partial_{\tau}\left(-\epsilon^{0}\right) & =-\left[\nabla \cdot \mathbf{e}-\partial_{\tau} \epsilon^{0}\right]=e j_{T}^{0} \\
\nabla \times \mathbf{h}-\left(-\partial_{0}\right)(-\mathbf{e})-\partial_{\tau} \epsilon & =\nabla \times \mathbf{h}-\partial_{0} \mathbf{e}-\partial_{\tau} \epsilon=e \mathbf{j}_{T} \\
\nabla \cdot \epsilon+\left(-\partial_{0}\right)\left(-\epsilon^{0}\right) & =\nabla \cdot \epsilon+\partial_{0} \epsilon^{0}=e j_{T}^{5} \\
\nabla \cdot \mathbf{h} & =0 \\
\nabla \times(-\mathbf{e})+\left(-\partial_{0}\right) \mathbf{h} & =-\left[\nabla \times \mathbf{e}+\partial_{0} \mathbf{h}\right]=0 \\
\nabla \times \epsilon-\sigma \partial_{\tau} \mathbf{h} & =0 \\
\nabla\left(-\epsilon^{0}\right)+\sigma \partial_{\tau}(-\mathbf{e})+\left(-\partial_{0}\right) \epsilon & =-\left[\nabla \epsilon^{0}+\sigma \partial_{\tau} \mathbf{e}+\partial_{0} \epsilon\right]=0
\end{aligned}
$$

which are form invariant under the choices

$$
\begin{aligned}
j_{T}^{0}\left(t_{T}, \mathbf{x}_{T}, \tau\right) & =-j^{0}(t, \mathbf{x}, \tau) \\
\mathbf{j}_{T}\left(t_{T}, \mathbf{x}_{T}, \tau\right) & =\mathbf{j}(t, \mathbf{x}, \tau) \\
j_{T}^{5}\left(t_{T}, \mathbf{x}_{T}, \tau\right) & =j^{5}(t, \mathbf{x}, \tau)
\end{aligned}
$$

From the transformation properties for the field strengths, we may deduce the transformation properties of the 5 -vector potential components. From

$$
f^{\alpha \beta}=\partial^{\alpha} a^{\beta}-\partial^{\beta} a^{\alpha}
$$

we have

$$
\mathbf{e}^{i}=\partial^{0} a^{i}-\partial^{i} a^{0} \underset{P}{\rightarrow}-\mathbf{e}^{i}=\partial^{0} a_{P}^{i}-\left(-\partial^{i}\right) a_{P}^{0}=-\left(\partial^{0} a^{i}-\partial^{i} a^{0}\right)
$$

so

$$
a_{P}^{0}=a^{0} \quad a_{P}^{i}=-a^{i}
$$

consistent with

$$
\mathbf{h}^{i}=\varepsilon^{i j k} \partial_{j} a_{k} .
$$

Similarly,

$$
\mathbf{e}^{i}=\partial^{0} a^{i}-\partial^{i} a^{0} \underset{T}{\rightarrow}-\mathbf{e}^{i}=\left(-\partial^{0}\right) a_{T}^{i}-\partial^{i} a_{T}^{0}=-\left(\partial^{0} a^{i}-\partial^{i} a^{0}\right)
$$

so

$$
a_{T}^{0}=-a^{0} \quad a_{T}^{i}=a^{i}
$$


again consistent with (117). For the second vector field,

$$
\epsilon^{i}=\partial^{5} a^{i}-\partial^{i} a^{5} \underset{P}{\rightarrow}-\epsilon^{i}=\partial^{5} a_{P}^{i}-\left(-\partial^{i}\right) a_{P}^{5}=-\left(\partial^{5} a^{i}-\partial^{i} a^{5}\right)
$$

leads to

$$
a_{P}^{5}=a^{5} \quad a_{P}^{i}=-a^{i}
$$

which is consistent with

$$
\epsilon^{0}=\partial^{5} a^{0}-\partial^{0} a^{5}
$$

and

$$
\epsilon^{i}=\partial^{5} a^{i}-\partial^{i} a^{5}
$$

Similarly

$$
\epsilon^{i}=\partial^{5} a^{i}-\partial^{i} a^{5} \underset{T}{\rightarrow} \epsilon^{i}=\partial^{5} a_{T}^{i}-\left(\partial^{i}\right) a_{T}^{5}=\left(\partial^{5} a^{i}-\partial^{i} a^{5}\right)
$$

requires

$$
a_{T}^{5}=a^{5} \quad a_{T}^{i}=a^{i}
$$

All of the 5-vector quantities encountered up to this point transform tensorially under space and time inversion, as the quantity $\left(x^{0}, \mathbf{x}, \tau\right)$. We summarize the results thus far in Table 1 ,

\begin{tabular}{|l|c|c|}
\hline \multicolumn{1}{|c|}{ Quantity } & Transformation Under $P$ & Transformation Under $T$ \\
\hline$\left(x^{0}, \mathbf{x}, \tau\right)$ & $\left(x^{0},-\mathbf{x}, \tau\right)$ & $\left(-x^{0}, \mathbf{x}, \tau\right)$ \\
\hline $\mathbf{e}$ & $-\mathbf{e}$ & $-\mathbf{e}$ \\
\hline $\mathbf{h}$ & $\mathbf{h}$ & $\mathbf{h}$ \\
\hline$\epsilon$ & $-\epsilon$ & $\epsilon$ \\
\hline$\epsilon^{0}$ & $\epsilon^{0}$ & $-\epsilon^{0}$ \\
\hline$\left(j^{0}, \mathbf{j}, j^{5}\right)$ & $\left(j^{0},-\mathbf{j}, j^{5}\right)$ & $\left(-j^{0}, \mathbf{j}, j^{5}\right)$ \\
\hline$\left(a^{0}, \mathbf{a}, a^{5}\right)$ & $\left(a^{0},-\mathbf{a}, a^{5}\right)$ & $\left(-a^{0}, \mathbf{a}, a^{5}\right)$ \\
\hline
\end{tabular}

Table 1 


\section{Off-Shell Quantum Mechanics}

We now turn to the discrete symmetries of the Schrodinger equation

$$
\begin{aligned}
\left(i \partial_{\tau}+e_{0} a_{5}\right) \psi(x, \tau) & =\frac{1}{2 M}\left(p^{\mu}-e_{0} a^{\mu}\right)\left(p_{\mu}-e_{0} a_{\mu}\right) \psi(x, \tau) \\
& =-\frac{1}{2 M}\left(\partial^{\mu}-i e_{0} a^{\mu}\right)\left(\partial_{\mu}-i e_{0} a_{\mu}\right) \psi(x, \tau)
\end{aligned}
$$

In the space reversed coordinates, the transformed equation satisfies

$$
\left(i \partial_{\tau}+e_{0} a_{5 P}\right) \psi_{P}\left(x_{P}, \tau\right)=-\frac{1}{2 M}\left(\partial_{P}^{\mu}-i e_{0} a_{P}^{\mu}\right)\left(\partial_{\mu P}-i e_{0} a_{\mu P}\right) \psi_{P}\left(x_{P}, \tau\right) .
$$

From Table 1 - and the fact that $\left(\partial_{0}, \partial_{k}, \partial_{\tau}\right)$ transforms as $\left(x_{0}, x_{k}, x_{5}\right)$ - we have

$$
\begin{aligned}
\left(i \partial_{\tau}+e_{0} a_{5}\right) \psi^{P}\left(x_{P}, \tau\right)= & \\
= & -\frac{1}{2 M}\left[\left(\partial_{P}^{k}-i e_{0} a_{P}^{k}\right)\left(\partial_{k P}-i e_{0} a_{k P}\right)\right. \\
& \left.\quad-\left(\partial_{P}^{0}-i e_{0} a_{P}^{0}\right)\left(\partial_{0 P}-i e_{0} a_{0 P}\right)\right] \psi^{P}\left(x_{P}, \tau\right) \\
= & -\frac{1}{2 M}\left[\left(-\partial^{k}+i e_{0} a^{k}\right)\left(-\partial_{k}+i e_{0} a_{k}\right)\right. \\
& \left.\quad-\left(\partial^{0}-i e_{0} a^{0}\right)\left(\partial_{0}-i e_{0} a_{0}\right)\right] \psi^{P}\left(x_{P}, \tau\right) \\
= & -\frac{1}{2 M}\left(\partial^{\mu}-i e_{0} a^{\mu}\right)\left(\partial_{\mu}-i e_{0} a_{\mu}\right) \psi^{P}\left(x_{P}, \tau\right)
\end{aligned}
$$

Since equation (132) is explicitly identical in form to (127), the solutions must be identical, so

$$
\psi_{P}\left(x_{P}, \tau\right)=\psi(x, \tau) \quad \Rightarrow \quad \psi_{P}(x, \tau)=\psi\left(x^{0},-\mathbf{x}, \tau\right)
$$

The corresponding argument for time inversion leads to

$$
\psi_{T}\left(x_{T}, \tau\right)=\psi(x, \tau) \quad \Rightarrow \quad \psi_{T}(x, \tau)=\psi\left(-x^{0}, \mathbf{x}, \tau\right)
$$

and we see that the manifest invariance under space and time inversion found for the HorwitzPiron theory with standard Maxwell fields 9] applies in the presence of pre-Maxwell fields. Seeking a solution for the replacement $e_{0} \rightarrow-e_{0}$, that is

$$
\left(i \partial_{\tau}-e_{0} a_{5}\right) \psi_{C}(x, \tau)=-\frac{1}{2 M}\left(\partial^{\mu}+i e_{0} a^{\mu}\right)\left(\partial_{\mu}+i e_{0} a_{\mu}\right) \psi_{C}(x, \tau)
$$

the usual strategy for Schrodinger-like equations with minimal coupling to a gauge field begins with complex conjugation, which expresses the antiunitary character of the total 
reflection discussed in the previous chapter. In the present case, complex conjugation of (127) leads to

$$
\left(-i \partial_{\tau}-e_{0} a_{5}\right) \psi^{*}(x, \tau)=-\frac{1}{2 M}\left(\partial^{\mu}+i e_{0} a^{\mu}\right)\left(\partial_{\mu}+i e_{0} a_{\mu}\right) \psi^{*}(x, \tau)
$$

which is not yet in the form (135). Unlike the case of Maxwell fields, the possible dependence of the pre-Maxwell fields on $\tau$ prevents us from simply taking $\tau \rightarrow-\tau$ in order to reverse the sign of $-i \partial_{\tau}$. Instead, we posit the existence of a $\tau$-inversion operation $\mathcal{T}$ and investigate the requirements which make it reasonable.

Applying $\tau$-inversion to (136), we find

$$
\left(i \partial_{\tau}-e_{0} a_{5 \mathcal{T}}\right) \psi^{*}(x,-\tau)=-\frac{1}{2 M}\left(\partial^{\mu}+i e_{0} a_{\mathcal{T}}^{\mu}\right)\left(\partial_{\mu}+i e_{0} a_{\mu \mathcal{T}}\right) \psi^{*}(x,-\tau)
$$

which will be in the form of (135) if

$$
\begin{aligned}
& a_{\mathcal{T}}^{\mu}\left(x_{\mathcal{T}}, \tau_{\mathcal{T}}\right)=a^{\mu}(x, \tau) \quad \Rightarrow \quad a_{\mathcal{T}}^{\mu}(x, \tau)=a^{\mu}(x,-\tau) \\
& a_{5 \mathcal{T}}\left(x_{\mathcal{T}}, \tau_{\mathcal{T}}\right)=-a_{5}(x, \tau) \quad \Rightarrow \quad a_{5 \mathcal{T}}(x, \tau)=-a_{5}(x,-\tau)
\end{aligned}
$$

Under the combination of transformations

$$
\begin{aligned}
\psi(x, \tau) \underset{C}{\longrightarrow} \psi_{C}(x, \tau) & =\psi^{*}(x,-\tau) \\
\tau \underset{C}{\longrightarrow} \tau_{C} & =-\tau \\
a^{\mu}(x, \tau) \underset{C}{\longrightarrow} a_{C}^{\mu}(x, \tau) & =a^{\mu}(x,-\tau) \\
a^{5}(x, \tau) \underset{C}{\longrightarrow} a_{C}^{5}(x, \tau) & =-a^{5}(x,-\tau)
\end{aligned}
$$

if they can be made consistent with the pre-Maxwell equations, the charge conjugate solution is $\psi_{C}(x, \tau)=\psi^{*}(x,-\tau)$.

To check the consistency of the transformations (141) to (143), we first find the 3-vector field strengths

$$
\begin{aligned}
& e^{k}=f^{0 k}=\partial^{0} a^{k}-\partial^{k} a^{0} \quad \underset{C}{\longrightarrow} e^{k} \\
& h^{k}=\varepsilon^{k i j} \partial_{i} a_{j} \quad \underset{C}{\longrightarrow} h^{k} \\
& \epsilon^{k}=f^{5 k}=\sigma \partial_{\tau} a^{k}-\partial^{k} a_{5} \quad \underset{C}{\longrightarrow}-\epsilon^{k} \\
& \epsilon^{0}=f^{50}=\sigma \partial_{\tau} a^{0}-\partial^{0} a_{5} \quad \underset{C}{\longrightarrow}-\epsilon^{0} \text {. }
\end{aligned}
$$


We now consider the Lorentz force equations

$$
\begin{aligned}
& M \frac{d^{2} x^{0}}{d \tau^{2}}=e_{0}\left[\mathbf{e} \cdot \frac{d \mathbf{x}}{d \tau}-\sigma \epsilon^{0}\right] \\
& M \frac{d^{2} \mathbf{x}}{d \tau^{2}}=e_{0}\left[\mathbf{e} \frac{d x^{0}}{d \tau}+\frac{d \mathbf{x}}{d \tau} \times \mathbf{h}-\sigma \epsilon\right]
\end{aligned}
$$

which become

$$
\begin{aligned}
& M \frac{d^{2} x_{C}^{0}}{d \tau^{2}}=e_{0}\left[\mathbf{e}_{C} \cdot\left(\frac{d \mathbf{x}}{d \tau}\right)_{C}-\sigma \epsilon_{C}^{0}\right] \\
& M \frac{d^{2} \mathbf{x}_{C}}{d \tau^{2}}=e_{0}\left[\mathbf{e}_{C}\left(\frac{d x^{0}}{d \tau}\right)_{C}+\left(\frac{d \mathbf{x}}{d \tau}\right)_{C} \times \mathbf{h}_{C}-\sigma \epsilon_{C}\right]
\end{aligned}
$$

so that

$$
\begin{aligned}
M \frac{d^{2} x^{0}}{d \tau^{2}} & =e_{0}\left[\mathbf{e} \cdot\left(-\frac{d \mathbf{x}}{d \tau}\right)-\sigma\left(-\epsilon^{0}\right)\right] \\
M \frac{d^{2} \mathbf{x}}{d \tau^{2}} & =e_{0}\left[\mathbf{e}\left(-\frac{d x^{0}}{d \tau}\right)+\left(-\frac{d \mathbf{x}}{d \tau}\right) \times \mathbf{h}-\sigma(-\epsilon)\right]
\end{aligned}
$$

and finally

$$
\begin{aligned}
& M \frac{d^{2} x^{0}}{d \tau^{2}}=-e_{0}\left[\mathbf{e} \cdot \frac{d \mathbf{x}}{d \tau}-\sigma \epsilon^{0}\right] \\
& M \frac{d^{2} \mathbf{x}}{d \tau^{2}}=-e_{0}\left[\mathbf{e} \frac{d x^{0}}{d \tau}+\frac{d \mathbf{x}}{d \tau} \times \mathbf{h}-\sigma \epsilon\right]
\end{aligned}
$$

Equations (154) and (155) are just the Lorentz equations (64) and (65) under the substitution $e_{0} \rightarrow-e_{0}$. Thus, while form invariance under $\tau$-inversion is not a reasonable symmetry to expect in pre-Maxwell theory and was not considered along with space and time reversal symmetry, we find that the classical operations

$$
\tau \rightarrow-\tau \quad a_{5} \rightarrow-a_{5}
$$

associated with quantum charge conjugation lead to a classical charge conjugation operation. The action of this charge conjugation on the field equations is

$$
\begin{gathered}
\nabla \cdot \mathbf{e}-\left(-\partial_{\tau}\right)\left(-\epsilon^{0}\right)=e j_{C}^{0} \\
\nabla \cdot \mathbf{e}-\partial_{\tau} \epsilon^{0}=e j_{C}^{0} \Rightarrow j_{C}^{0}=j^{0} \\
\nabla \times \mathbf{h}-\partial_{0} \mathbf{e}-\left(-\partial_{\tau}\right)(-\epsilon)=e \mathbf{j}_{C} \\
\nabla \times \mathbf{h}-\partial_{0} \mathbf{e}-\partial_{\tau} \epsilon=e \mathbf{j}_{C} \Rightarrow \mathbf{j}_{C}=\mathbf{j}
\end{gathered}
$$




$$
\begin{gathered}
\nabla \cdot(-\epsilon)+\partial_{0}\left(-\epsilon^{0}\right)=e j_{C}^{5} \\
\nabla \cdot \epsilon+\partial_{0} \epsilon^{0}=-e j_{C}^{5} \Rightarrow j_{C}^{5}=-j^{5} \\
\nabla \cdot \mathbf{h}=0 \\
\nabla \times \mathbf{e}+\partial_{0} \mathbf{h}=0 \\
\nabla \times(-\epsilon)-\sigma\left(-\partial_{\tau}\right) \mathbf{h}=0 \\
\nabla \times \epsilon-\sigma \partial_{\tau} \mathbf{h}=0 \\
\nabla\left(-\epsilon^{0}\right)+\sigma\left(-\partial_{\tau}\right) \mathbf{e}+\partial_{0}(-\epsilon)=0 \\
\nabla \epsilon^{0}+\sigma \partial_{\tau} \mathbf{e}+\partial_{0} \epsilon=0
\end{gathered}
$$

and so we see that, taking the action on the currents as

$$
\left(j^{0}, \mathbf{j}, j^{5}\right) \underset{C}{\longrightarrow}\left(j^{0}, \mathbf{j}, j^{5}\right)_{C}=\left(j^{0}, \mathbf{j},-j^{5}\right) \quad,
$$

the pre-Maxwell equations transform consistently under the action of classical charge conjugation. Moreover, we find that current conservation

$$
\partial_{\mu} j^{\mu}+\partial_{\tau} j^{5}=0 \quad \underset{C}{\longrightarrow} \partial_{\mu} j^{\mu}+\left(-\partial_{\tau}\right)\left(-j^{5}\right)=0
$$

is preserved. In quantum mechanics, the current $j^{5}$ is interpreted as the probability of finding a particle in a localized volume of space time at a given $\tau$, and the meaning of $j_{C}^{5}=-j^{5}$ must be examined carefully.

\section{Conclusions}

The standard account of the discrete symmetries in quantum theory is deeply influenced by Wigner's prescription [4 for time reversal, which operates on both the coordinate time and the temporal ordering of events. It should be noted that Wigner was concerned (both in 1932 and 1959) with the non-relativistic quantum mechanics of atomic spectra, and his explicit use of Galilean time determined his notion of time reversal ${ }^{2}$. Thus, neither negative

\footnotetext{
${ }^{2}$ Wigner comments in 4], "Hence, 'reversal of the direction of motion' is perhaps a more felicitous, though longer, expression than 'time inversion.' "
} 
energies nor pair creation/annihilation played any part in his considerations. If, in the spirit of Stueckelberg, we wish to disentangle the symmetries of the coordinate time $t$ from those of the temporal parameter $\tau$, then we expect that the discrete reflections will lead to the following interpretations:

1. Space inversion covariance $P$ implies certain symmetric relations between a given experiment and one performed in a spacially reversed configuration.

2. Time inversion covariance $T$ implies certain symmetric relations between a given experiment and one performed in a time-reversed configuration, which is to say one in which advancement in $t$ is replaced by retreat, and so a trajectory with $\dot{x}^{0}>0$ is replaced by a trajectory with $\dot{x}^{0}<0$. Thus, we expect symmetric behavior between pair annihilation processes and pair creation processes.

3. Charge conjugation covariance $C$ implies certain symmetric relations between a given experiment and one in which the events are traced out in the reverse order and carry opposite charge. Applying (55) to this case, we expect that the additive charges associated with internal symmetries also undergo inversion.

The analysis of the discrete symmetries in the Stueckelberg formalism — extended to include the parameter $\tau$ in local gauge group — demonstrates the theory's form invariance under spacetime inversion, and exposes the charge conjugation symmetry, leading naturally to a view of these symmetries based on interpretations 1 to 3 above. The structure of the classical electromagnetic theory requires that, unlike the case for the Maxwell field, the off-shell gauge field behave tensorially under the discrete Lorentz transformations $P$ and $T$. Given these conditions on the gauge fields, the quantum theory is seen to be invariant in a very simple way under space and time reversal, and we may identify interpretations 1 and 2 - the space inversion operation $P$ exchanges a particle trajectory with its mirror image, and the time inversion operation $T$ exchanges particle trajectories with antiparticle trajectories.

On the other hand, we do not regard the charge conjugation operation as connecting symmetrical dynamical evolutions. The requirement that solutions exist for the charge reversed case leads to an operation which reverses $x^{5}=\tau$ and the corresponding fifth-component 
objects, $a^{5}$ and $j^{5}$. Thus, the resulting charge conjugation operation, reverses the temporal order of events and the sign of the charge and related currents, leading to a negative probability density

$$
j^{5}=|\psi(x, \tau)|^{2} \underset{C}{\longrightarrow} j_{C}^{5}=-j^{5},
$$

which only makes sense in the context of the current conservation expression (170). Rather we associate the reversal of temporal order performed by charge conjugation with the reordering of events performed by the observer in the laboratory, who interprets events as always evolving from earlier to later values of $t$. Thus, charge conjugation exchanges the viewpoint of the events under interaction with the viewpoint of the laboratory observer. The inversion of charges (associated with the gauge symmetry and any internal symmetries) under this exchange reinforces the conventional view of antiparticles in the laboratory, but does not influence the event dynamics. Following Stueckelberg, we return to a formalism of events interacting through gauge fields with events which may propagate equivalently with $d t / d \tau<0$ or $d t / d \tau>0$, and understand the antiparticle to simply be that part of an event trajectory for which $d t / d \tau<0$. The significance of the charge conjugation operation is that the reversal of quantum numbers is observed in the laboratory when the observer uses the laboratory clock as the parameter which orders the events.

In the context of Horwitz-Piron theory, the discrete symmetries of (8) were studied in [9]. That study, which assumed the standard transformation properties for the four-vector Maxwell potential

$$
\left(A^{0}, \mathbf{A}\right) \underset{P}{\rightarrow}\left(A^{0},-\mathbf{A}\right) \quad\left(A^{0}, \mathbf{A}\right) \underset{T}{\rightarrow}\left(A^{0},-\mathbf{A}\right)
$$

similarly concluded that while events interact without concern for the particle/antiparticle distinction, the $C P T$ conjugate of the negative energy trajectory is observed in the laboratory as the antiparticle.

A field theoretic study of the discrete symmetries and their significance in quantum scattering will be reported in a forthcoming paper. 


\section{References}

[1] E. C. G. Stueckelberg, Helv. Phys. Acta 14 (1941) 322,

E. C. G. Stueckelberg, Helv. Phys. Acta 14 (1941) 588.

[2] V. A. Fock, Phys. Z. Sowjetunion 12 (1937) 404.

[3] L. P. Horwitz, R. I. Arshansky and A. C. Elitzur, Found. of Phys. 18 (1988) 1159.

[4] Eugene P. Wigner, Group Theory and its Application to the Quantum Mechanics of Atomic Spectra, Academic Press, NY, 1959,

E. Wigner, Nachr. Akad. Ges. Wiss. Göttingen 31 (1932) 546.

[5] C. Itzykson J.-B. Zuber, Quantum Field Theory, McGraw-Hill, New York, 1980.

[6] R. P. Feynman, Phys. Rev. 80 (1950) 440.

[7] A. Kyprianidis, Phys. Rep. 155 (1987) 1.

[8] M. C. Land and L. P. Horwitz, Found. of Phys. Lett. 4 (1991) 61.

[9] R. Arshansky, L. P. Horwitz and Y. Lavie, Found. of Phys. 13 (1983) 1167.

[10] L. P. Horwitz and C. Piron, Helv. Phys. Acta 48 (1973) 316.

[11] L. P. Horwitz and Y. Lavie,Phys. Rev. D 26 (1982) 819

R. I. Arshansky and L. P. Horwitz, J. Math. Phys. 30 (1989) 213,

R. I. Arshansky and L. P. Horwitz, Phys. Lett A 131 (1988) 222.

[12] R. Arshansky and L. P. Horwitz, J. Math. Phys. 30 (1989) 66.

[13] R. Arshansky and L. P. Horwitz, J. Math. Phys. 30 (1989) 380.

[14] M. C. Land, R. Arshansky, and L. P. Horwitz, Found. of Phys. 24 (1994) 563.

[15] M. C. Land and L. P. Horwitz, J. Phys. A: Math. and Gen. 28 (1995) 3289.

[16] M. C. Land, Found. of Phys. 31 (2001) 967. 
[17] M. C. Land, N. Shnerb, L. P. Horwitz, J. Math. Phys. 36 (1995) 3263, F. J. Dyson, Am. J. Phys. 58 (1990) 209, Shogo Tanimura, Ann. Phys. 220 (1992) 229.

[18] M. C. Land and L. P. Horwitz, Found. of Phys. 21 (1991) 299.

[19] D. Saad, L. P. Horwitz and R. I. Arshansky, Found. of Phys. 19 (1989) 1126.

[20] M. C. Land, Found. of Phys. 27 (1996) 19.

[21] M. C. Land, Found. of Phys. 33 (2003) 1157.

[22] Yu. V. Novozhilov, Introduction to Elementary Particle Theory, Pergamon Press, Oxford, 1975.

[23] J. D. Jackson, Classical Electrodynamics, John Wiley and Sons, NY, 1975. 